\title{
Statistical description of sediment transport experiments
}

\author{
Christophe Ancey \\ École Polytechnique Fédérale de Lausanne, Ecublens, 1015 Lausanne, Switzerland \\ Tobias Böhm \\ Cemagref, Domaine Universitaire, BP 76, 38402 Saint-Martin-d'Hères Cedex, France
}

Magali Jodeau

Cemagref, 3 bis quai Chauveau, 69336 Lyon, France

Philippe Frey

Cemagref, Domaine Universitaire, BP 76, 38402 Saint-Martin-d'Hères Cedex, France

(Received 28 December 2005; revised manuscript received 14 March 2006; published 18 July 2006)

\begin{abstract}
A longstanding problem in the study of sediment transport in gravel-bed rivers is related to the physical mechanisms governing bed resistance and particle motion. To study this problem, we investigated the motion of coarse spherical glass beads entrained by a steady shallow turbulent water flow down a steep twodimensional channel with a mobile bed. This experimental facility is the simplest representation of sediment transport on the laboratory scale, with the tremendous advantages that boundary conditions are perfectly controlled and a wealth of information can be obtained using imaging techniques. Flows were filmed from the side by a high-speed camera. Using image processing software made it possible to determine the flow characteristics such as particle trajectories, their state of motion (rest, rolling, or saltating motion), and flow depth. In accordance with earlier investigations, we observed that over short time periods, sediment transport appeared as a very intermittent process. To interpret these results, we revisited Einstein's theory on sediment and derived the statistical properties (probability distribution and autocorrelation function) of the key variables such as the solid discharge and the number of moving particles. Analyzing the autocorrelation functions and the probability distributions of our measurements revealed the existence of long-range correlations. For instance, whereas theory predicts a Binomial distribution for the number of moving particles, experiments demonstrated that a negative binomial distribution best fit our data, which emphasized the crucial role played by wide fluctuations. These frequent wide fluctuations stemmed particle entrainment and motion being collective phenomena rather than individual processes, contrary to what is assumed in most theoretical models.
\end{abstract}

DOI: 10.1103/PhysRevE.74.011302

PACS number(s): 45.70.Mg, 45.50.-j, 47.85.-g, 92.10.Wa

\section{INTRODUCTION}

The objective of this paper is to describe the statistical properties of experiments on two-phase flows involving coarse particles driven by gravity and drag exerted by a water turbulent flow. This issue is closely related to the microstructural understanding of particle transport in waterways or industrial pipelines. Despite substantial progress made over the last two decades in the physical understanding of the motion of coarse particles in a turbulent stream, the ability to compute bulk quantities such as the sediment flux in rivers remains poor. For instance, the sediment flow rates measured in gravel-bed rivers differ within one to two orders of magnitude from the bed-load transport equations [1-3], even though these equations have been established from flume experiments using regression techniques and are believed to provide a proper evaluation of sediment transport in a wellcontrolled laboratory environment. Surprisingly enough, simple power-law models relating the sediment flow rate to the water flow depth can perform better than more sophisticated physically based models [3].

At first glance, this situation may be seen as paradoxical since experiments usually reveal a simple behavior, e.g., a linear or pseudolinear dependence of the solid discharge on the water discharge. Figure 1 shows the typical linear trends that we observed with our experimental setup. Similar trends were also obtained with alternative experiments in long flumes and field data $[4,5]$. This apparent simplicity, however, challenges our capacity of providing simple analytical explanations.

Impediments to a full analytical approach to two-phase flows are many: complex interplay between the particles and

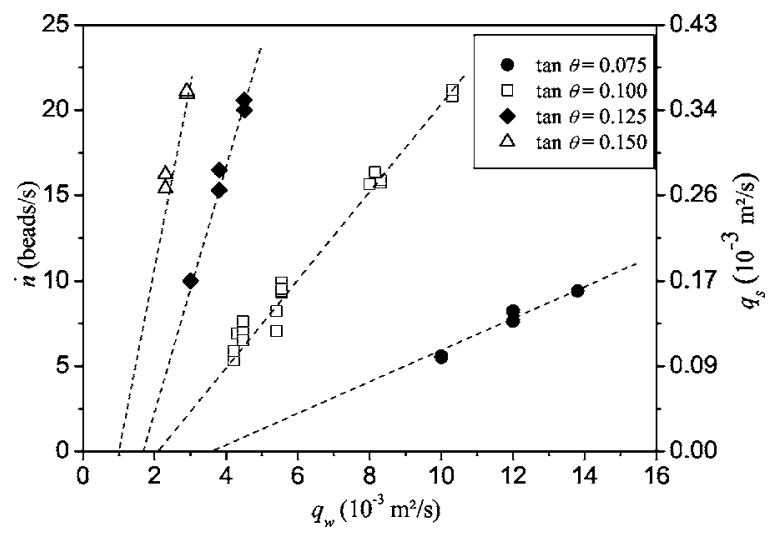

FIG. 1. Solid discharge (volume flow rate $q_{w}$ or flux of beads $\dot{n}$ ) as a function of the water discharge for different channel slopes $\theta$ in our flume. 
the carrying fluid, particle exchanges between the bed and the flow, turbulence effects (bed friction, advection of turbulent structures), etc. That is why most models are based on substantial approximations of the interplay between the solid and fluid phases. In the field of bed-load transport, the meanfield approximation primarily proposed by Bagnold $[6,7]$ is a pervasive assumption. According to this hypothesis, the fluid shear stress at the bed $\tau_{0}$ equals the threshold value $\tau_{c}$ corresponding to incipient motion of particles; the difference between the total bottom shear stress $\tau$ and $\tau_{0}$ provides the shear stress in the solid phase. Interpreting the latter stress as the result of momentum transfers between the solid and fluid phases makes it possible to theoretically compute the number of particles that can be entrained and maintained in motion, and thereby the solid flow rate (or solid discharge) [8]. This type of approximation leads to a solid discharge $q_{s}$ in the form $q_{s} \propto\left(\tau-\tau_{c}\right)^{3 / 2}$. When compared with laboratory experimental data or field measurements, this scaling correctly describes the sediment transport for steady uniform or gently varying flows at sufficiently high solid discharges [9]. There are, however, a number of clues that indicate that Bagnold's formulation may be flawed or crude. First, for this scaling to match experimental observations, the bulk particle friction coefficient introduced by Bagnold must be fit to unphysical values $[10,11]$. Second, for flow conditions that depart from steady uniform flow conditions, Bagnold's approximation yields poor results, notably for flows over arbitrarily sloping beds [12] or at low levels of solid discharge $[10,13]$. Third, if bedforms (dune or antidune depending on the value of the Froude number) are interpreted as resulting from a loss of linear stability in the coupled fluid-solid system, the Bagnold assumption fails to capture the necessary physics since the resulting equations of motion do not show any instability [14].

From the physical point of view, Bagnold's approach takes its origin in the strong belief that sediment transport results from an equilibrium in the momentum transfer between solid and liquid phases. The mean-field treatment applied by Bagnold conflicts with a noticeable feature of sediment transport: its intermittent nature at low flow rates. In his simplified fluid-mechanics treatment, Bagnold averaged the temporal and spatial variability of near-bed turbulence to derive an averaged momentum transfer between the fluid and solid phases. However, both laboratory and field measurements reveal that particle movement occurs for a period of time followed by a period in which no motion occurs [15-17]. At low flow rates, intermittent motion occurs when the near-bed downstream velocity is sufficiently high to destabilize stationary particles and set them in motion. This situation corresponds to instances in which the instantaneous near-bed velocity outweighs its mean value as a result of near-bed turbulence and does not correlate well with the mean flow conditions far from the bed $[13,18]$.

In contrast to Bagnold, Einstein realized how important it is to account for the episodic nature of particle transport in computing the solid discharge [19]. In Einstein's view, sediment transport does not result from an equilibrium in the momentum transfers between solid and liquid phases, but rather from the difference between the entrainment and deposition rates, $E$ and $D$, respectively, which are a function of the flow conditions and bed geometry. Stated more explicitly, this amounts to writing that on a small interval $\delta x$, the solid discharge variation is $\delta q_{s}=(E-D) \delta x$. The solid discharge at bed equilibrium is the implicit solution to the equation $E$ $=D$. Einstein's stochastic approach raises a number of issues that have received few responses to date. For instance, since particles move sporadically and in different groups, the solid flow rate is made up of a series of pulses and is highly fluctuating, which makes it difficult to define and measure it properly, even under steady flow conditions [20]. Both field and laboratory experiments have revealed that instances in which the instantaneous solid discharge is four times higher than its mean value are frequent [21-23]. Translated statistically, this observation means that the probability density functions of the transport-rate records have a thick tail and depart from the expected Gaussian behavior. This departure can be seen as the hallmark of collective motions [24]; if so, this also implies that any mean-field approximation runs into difficulty since cooperation between particles is not accounted for.

This paper aims to gain insight into the statistical properties of idealized sediment-transport experiments. Fundamentally, we note that every bed-load transport model must use ad hoc parameters adjusted on experimental data. This recourse to fitting is widespread and likely unavoidable given the complexity of the physical processes involved. However, this practice makes it difficult to test the reliability and performance of a physically based model and, in a sense, blurs the physical picture drawn from experiments by enforcing agreement. Testing model efficiency must then be carried out using alternative tools. Here our claim is that analyzing the statistical properties of measured and predicted variables offers a more robust and stringent framework than the usual comparison between predicted and measured quantities.

Our idea was to run experiments in an inclined, twodimensional flume with a continuous particle supply and steady flow rate. This two-dimensional flume is assumed to be the simplest representation of sediment transport on the laboratory scale and presents overwhelming advantages: the boundary conditions can be controlled and most of the flow variables can be measured using image processing. Since a quantitative comparison between theory and experiment is biased by any parameter fitting, we tested theory by analyzing the probability distributions and correlations of the signals measured. Here we have considered Einstein's theory as the prototype of theories prone to probabilistic interpretation. In Sec. II, we will reformulate the original formulation given by Einstein [19] to make the physical arguments more apparent. In Sec. III, we will present the experimental facilities and procedures. Finally, in Sec. IV, we will interpret our results from the probabilistic point of view. A short video clip showing a typical experiment is available as an EPAPS document [25].

\section{EINSTEIN'S THEORY REVISITED}

Using ad hoc arguments, Einstein derived a bed-load equation, which has been considered as the cornerstone of probabilistic theories of bed-load transport (see Ref. [19], pp. 


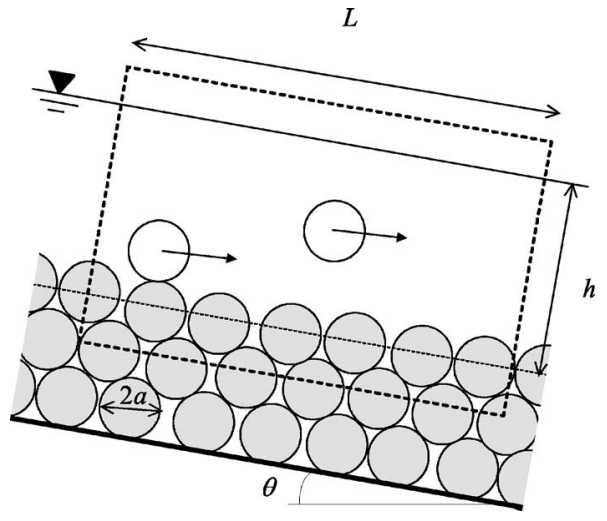

FIG. 2. Sketch defining the flow configuration.

29-38). Taking inspiration from the work done by Lisle et al. [26] and Papanicolaou et al. [27], we assume that sediment transport at low flow rates can be described using a birthdeath process. Essentially we are trying to generalize Einstein's theory and to cast it in more physical terms and derive the statistical properties of the key variables. After specifying notations (Sec. II A), we will present a generalized Einsteintype model (Sec. II B). The Appendix outlines Einstein's original method for computing the sediment-transport rate.

\section{A. Objective and notations}

We consider a two-dimensional, steady water stream flowing down a bed, the mean slope of which is denoted by $\theta$. The bed is made up of mobile, spherical particles of equal radius $a$ and density $\rho_{p}$. The water flow rate $q_{w}$ is prescribed at the channel entrance. In the meantime, the flume is supplied with particles identical to those making up the bed in such a way that there is bed-load equilibrium, i.e., on average over long time intervals, there is neither erosion nor deposition along the bed. Figure 2 provides a sketch of the flow configuration.

A few solid particles are entrained by the water stream: the particles can roll/slide along the bed or they can leap and stay in saltation in the water stream for short periods of time. We refer to the former motion as the rolling regime and to the latter as the saltating regime. Since we are especially interested in weakly intense bed-load transport, emphasis is given to flows with a fairly low fluid velocity. In this case, the trajectory of one particle exhibits a succession of rests and moves in a rolling or saltating regime, which may make it difficult to accurately discriminate the motion regime. For the sake of simplicity, we will assume here that there is only one species of moving particles, which is referred to as the moving (i.e., saltating and rolling) particles $M$.

In a typical bed-load transport problem, knowing the control parameters of this flow configuration $\left(q_{w}, \theta, a, \rho_{p}\right)$, we wish to determine the flow properties: flow depth $h$, solid discharge $q_{s}$ (number of particles per unit time conveyed by the flow, measured in $\mathrm{m}^{3} / \mathrm{s}$ ), and mean flow $\bar{u}_{f}$. For this purpose, we consider a control volume $\mathcal{V}$, within which we follow up the particles and the fluid motion; we pose $V=\mathcal{S}$ $\times L$, where the length is denoted by $L$, the volume by $V$, the cross section by $\mathcal{S}=h W$, where $W$ represents the flume width. Here, instead of focusing on the relationships between the mean values, we seek the statistical properties of the key quantities such as the solid discharge.

\section{B. Birth-death process as a generalization of Einstein's model}

The solid discharge can be defined as the flux of particles through a flow cross section $\mathcal{S}: q_{s}=\int_{\mathcal{S}} \mathbf{u}_{p} \cdot \mathbf{k} d \mathcal{S}$, where $\mathbf{k}$ is the unit normal to $\mathcal{S}$. This definition, suitable for continuum fields, is not well suited to discrete elements. A more convenient definition is to introduce the flow rate in terms of the probability $P\left[\mathbf{u}_{p} \mid \mathbf{x}, t\right]$ that a particle crosses the control surface $\mathcal{S}$ at position $\mathbf{x}$ and time $t$ with velocity $\mathbf{u}_{p}$,

$$
q_{s}=\int_{\mathcal{S}} \int_{\mathbb{R}^{2}} P\left[\mathbf{u}_{p} \mid \mathbf{x}, t\right] \mathbf{u}_{p} \cdot \mathbf{k}|d \mathbf{x}| d \mathbf{u}_{p} .
$$

Under steady conditions $(\partial P / \partial t=0)$, this definition can be worked out as

$$
q_{s}=\lim _{V \rightarrow \infty} \frac{1}{V} \sum_{i=1}^{N} u_{i} v_{p} \mathcal{S}=\lim _{L \rightarrow \infty} \frac{v_{p}}{L} \sum_{i=1}^{N} u_{i},
$$

in which the ensemble average has been replaced by a volume average and $v_{p}$ is the particle volume. The integration has been made on the control volume $\mathcal{V}$. We have also used $u_{i}=\mathbf{u}_{p} \cdot \mathbf{k}$ the streamwise velocity component of particle $i$ and $n$ the number of particles in motion in the control volume. Therefore, in the following, we shall define the flow rate $\dot{n}$ $=q_{s} / v_{p}$ as

$$
\dot{n}=\frac{1}{L} \sum_{i=1}^{n} u_{i}
$$

In order to compute the discharge equation, we need to establish (i) the number $n$ of particles in motion and (ii) their velocities depending on the control parameters $\left(q_{w}, \theta, a, \rho_{p}\right)$.

In order to compute the number of particles in motion, we can draw an analogy with chemical reactions. If the particles resting on the bed surface are denoted by $B$, the moving particles by $M$, we can represent the exchanges between the two phases in the following way:

$$
B \rightleftarrows M .
$$

From these equations, we can establish a kinetic equation, which tells us the rate at which exchanges occur between the species $B$ and $M$. The time variation in the number of moving particles is

$$
\frac{d n}{d t}=\frac{n_{b \rightarrow m}}{t_{b}}-\frac{n_{m \rightarrow b}}{t_{m}},
$$

where $n_{b \rightarrow m}$ is the number of particles dislodged from the bed and $n_{m \rightarrow b}$ is the number of moving particles that are left to rest within the observation window. These population exchanges are associated with the characteristic times $t_{b}$ and $t_{m}$, which are in turn related to the mean times during which a single particle stays at rest or moves, respectively, $\sigma$ and $\tau$ [see Fig. 3(a)]. We can also use Eq. (4) to define the entrain- 

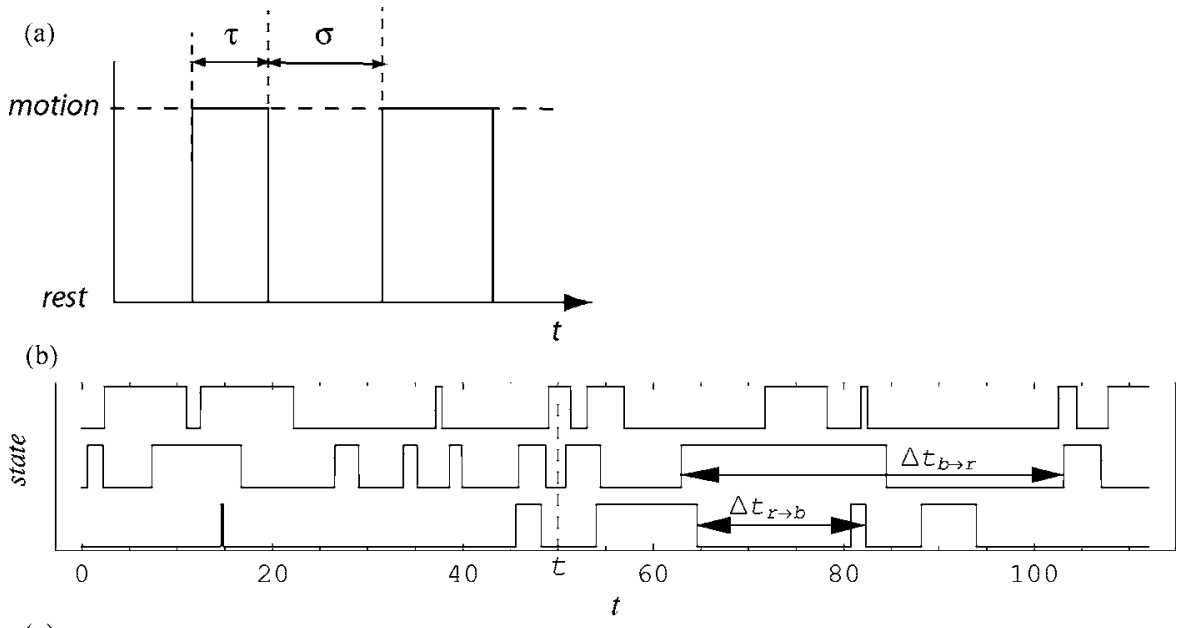

(c)

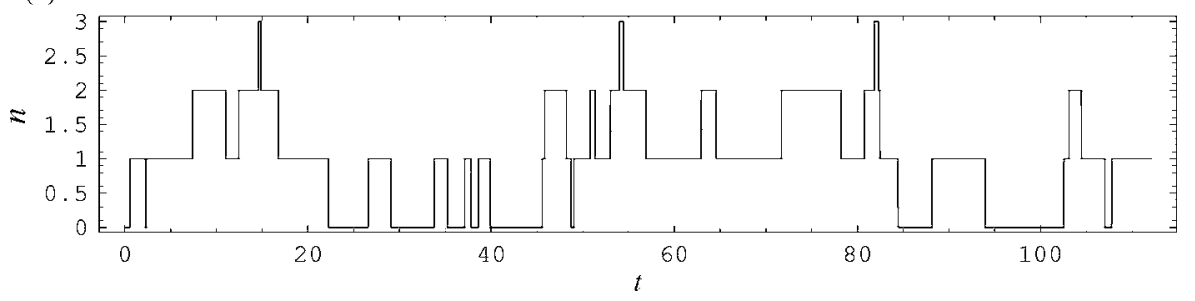

FIG. 3. (a) Succession of resting and moving phases for a single particle. (b) Superimposition of $N$ telegrapher's processes. (c) The number of moving particles is computed as the sum of the state variables; the waiting time for a single particle is defined as the time period elapsed between two events of the same type. Simulations made with $N$ $=3$ particles, $\tau=5 \mathrm{~s}$, and $\sigma=10 \mathrm{~s}$. ment rate (first term on the right-hand side) and the deposition rate (second on the right-hand side).

Transitions between the moving and/or resting states occur randomly. Following Lisle et al. [26] and Papanicolaou et al. [27], we assume that the particle motion is influenced only by its present state and has a fade memory of its previous states. In other words, the state transitions are governed by a continuous-time Markov process of order 1, with two discrete states (moving and/or resting). If we further assume that there is time and space invariance in the erosion and/or deposition process, the Markov transitions occur with constant probability per unit time. For any small time increment $\delta t$, we have

$\operatorname{Prob}($ moving at time $t+\delta t \mid$ resting at time $t)=\sigma^{-1} \delta t+o(\delta t)$,

Prob(resting at time $t+\delta t \mid$ moving at time $t)=\tau^{-1} \delta t+o(\delta t)$,

where the characteristic times $\sigma$ and $\tau$ are constant. This two-state Markov process is known as a telegrapher's process (see Ref. [28]). With these assumptions, it can be shown that the resting and moving times are exponentially distributed with means $\sigma$ and $\tau$, respectively. If $T_{b, i}$ and $T_{m, i}$ represent the durations of the $i$ th periods of rest and motion since observation has started, then $\operatorname{Prob}\left(T_{m}\right)=\tau^{-1} \exp \left(T_{m} / \tau\right)$ and $\operatorname{Prob}\left(T_{b}\right)=\sigma^{-1} \exp \left(T_{b} / \sigma\right)$; said differently, the waiting time $\Delta t_{b \rightarrow r}$ between two entrainments is exponentially distributed with a mean time equal to $\sigma, \operatorname{Prob}\left(\Delta t_{b \rightarrow r}\right)=(\sigma$ $+\tau)^{-1} \exp \left[\Delta t_{b \rightarrow r} /(\sigma+\tau)\right]$ (see Fig. 3). Using the correspondence between the Poisson and exponential distributions, we also deduce that the number of events (deposition and/or entrainment) that occur per unit time is distributed according to a Poisson distribution: the probability that we observe $k$ entrainments of the same particle within the time interval $\delta t$ (of any duration) is given by $\operatorname{Prob}(k ; \delta t)=\nu^{k} \exp ^{-\nu} / k$ !, with $\nu=\delta t / \sigma$. The autocorrelation function of a random variable $X$ being defined as

$$
\rho(s)=\frac{\langle[X(t+s)-\bar{X}][X(t)-\bar{X}]\rangle}{\left\langle[X(t)-\bar{X}]^{2}\right\rangle},
$$

where $\langle\cdot\rangle$ denotes time averaging and $\bar{X}$ the mean value, we find that the autocorrelation function for the telegrapher's process is $\rho(s)=\exp [-(1 / \tau+1 / \sigma) s][28]$.

This description is a simplified probabilistic Lagrangian description of a single particle's motion. We generalize it to obtain a Eulerian viewpoint, where we describe the motion of $n$ particles within an observation window. Within our observation window of length $L=2 \lambda a \gg a$ ( $\lambda$ being a free parameter that can take any value), we assume that on average, the particle flux is steady, which means that the particles that leave the window are replaced by other particles coming from upstream. Within this window, there are approximately $N=\lambda$ particles lying over the bed surface, either at rest or in motion. The number $n$ of particles in motion is then the sum of $N$ variables independently distributed and governed by a telegraph process [see Fig. 3(b)]. Figure 3(c) shows a typical variation in the number of moving particles; in that case, the number of moving particles is a step-shaped function inheriting the statistical properties of the individual processes.

Indeed, since each particle is governed by a telegraph process, the probability of observing it in motion is $\xi=\tau /(\tau$ $+\sigma)$, i.e., it follows a Bernoulli distribution. Now, summing $N$ particles following a Bernoulli distribution leads to a binomial distribution with mean $\bar{n}=\xi N$ and variance $\xi(1-\xi) N$. We then conclude that with our assumptions, the number $n$ of moving particles is distributed according to a binomial distribution, which means that if $\xi$ stays constant independently of the number of particles $N$ when $L \rightarrow \infty$, then the probabil- 
ity distribution of $n$ tends toward a Gaussian distribution. If we further assume that whenever a particle is set in motion, it reaches a fairly constant velocity $u_{p}$ [29], then using Eq. (3) leads to concluding that the probability distribution of the solid discharge is the binomial distribution $\mathrm{Bi}$, with mean $\xi N u_{p}$ and variance $\xi(1-\xi) N u_{p}^{2}$,

$$
\operatorname{Prob}(\dot{n})=\operatorname{Bi}\left[\xi N u_{p}, \xi(1-\xi) N u_{p}^{2}\right] .
$$

In the large $N$ limit, this distribution tends to be Gaussian. Since the sum of Poisson-distributed variables also has a Poisson distribution, we infer that the number of deposition and/or entrainment events per unit time has a Poisson distribution: the probability that we observe $k$ entrainments within the time interval $\delta t$ is given by

$$
\operatorname{Prob}(k ; \delta t)=\frac{\nu^{k}}{k !} \exp ^{-\nu},
$$

with $\nu=N \delta t / \sigma$. Instead of the Poisson distribution for characterizing the number of events per unit time, we can equivalently use the exponential distribution for specifying the lag times between two events; the mean waiting times between two entrainments within the observation window is $(\sigma$ $+\tau) / N$. The autocorrelation function of $N$ parallel telegrapher's processes is

$$
\rho(s)=\exp \left(-s / \tau_{*}\right),
$$

with $\tau_{*}=\sigma \tau /(\tau+\sigma) / N$. It is worth noting that, with our assumptions, the solid discharge and the number of moving particles have the same autocorrelation function.

According to Einstein [19], the probability of entrainment is the fraction of time $\xi=\tau /(\tau+\sigma)$ that a particle is in a moving state. It also represents, on average, the relative number of particles (i.e., $p$ ) that have moved within the observation window for a given time interval [27]. Moreover, in Einstein-type theories, particle entrainment results from a loss of stability: when the instantaneous lift and/or drag force exceeds the resisting forces, the particle is dislodged from the bed and starts to roll. By relating the fluid forces to the instantaneous fluid velocity $u_{f}$, we can deduce the fluid threshold $u_{c}$ corresponding to incipient motion (e.g., see Refs. $[27,30])$. The probability $p$ is then defined as $p=\xi$ $=\operatorname{Prob}\left(u_{f}>u_{c}\right)$. Over the time interval $t_{b}$, the number of particles that are entrained is then $n_{b \rightarrow m}=N p$, while the number of particles that come to a halt is $n_{m \rightarrow b}=n(1-p)$ over the period $t_{m}$. In steady flow conditions, Eqs. (4) and (3) lead to

$$
q_{s}=\frac{p}{1-p} \frac{t_{m}}{t_{b}} \frac{N}{L} u_{p}=\frac{p}{1-p} \frac{t_{m}}{t_{b}} \frac{u_{p}}{2 a},
$$

which is formally similar to Eq. (A1) derived by Einstein, except that the solid discharge is now explicitly dependent on the particle velocity.

Our goal is not to derive a complete discharge equation and so we do not go further by specifying the relation of $p$ to the mean flow conditions, as Einstein [19] or subsequent authors did. Here we focus on the main elements of his theory, i.e., the general expressions of the deposition and entrainment rates, the solid discharge, and the number of particles in motion. We have not only inferred the mean val- ues of these variables, but also their expected statistical distributions.

\section{EXPERIMENTAL FACILITIES AND TECHNIQUES}

\section{A. Overview}

In order to test the influence of fluid velocity on bed-load transport, we ran six experiments with different flow rates in a two-dimensional channel (see Sec. III B). The features of each run are summarized in Table I. The hydraulic conditions are specified using classic dimensionless numbers. The flow Reynolds number is defined as $\operatorname{Re}=4 R_{h} \bar{u}_{f} / \nu$, where $R_{h}$ $=W h /(2 h+W)$ denotes hydraulic radius, $\bar{u}_{f}=q_{w} /(W h)$ fluid velocity (averaged in the $y$ and $z$ directions), $\nu$ kinematic viscosity of water, and $h$ the time-averaged water depth. The Froude number $\mathrm{Fr}=\bar{u}_{f} / \sqrt{g h}$ varied significantly over the duration of the experiment and along the main stream direction. The mean Fr values are reported in Table I. The Shields number is defined as $N_{\mathrm{Sh}}=\rho_{f} \bar{u}_{f}^{2} /\left[\left(\rho_{p}-\rho_{f}\right) g h\right]$ and reflects the ratio of the water driving force to the friction resistance force on the bed [23]. The solid concentration is defined as the ratio of the solid and water discharges $C_{s}=q_{s} / q_{w}$. Values reported in Table I are low, which indicates that particle flow was dilute. The $h / d$ ratio is low, typically in the range $1.7-$ 3.2 .

\section{B. Channel}

Experiments were carried out in a tilted, narrow, glasssided channel, $2 \mathrm{~m}$ in length and $20 \mathrm{~cm}$ in height. Figure 4 shows a sketch of the experimental facility. The channel width $W$ was adjusted to $6.5 \mathrm{~mm}$, which was slightly larger than the particle diameter $(6 \mathrm{~mm})$. In this way, particle motion was approximately two dimensional and stayed in the focal plane of the camera (see Sec. III E and Sec. III F). The channel slope $\tan \theta$ was $10 \%$.

\section{Channel base and mobile bed}

The channel base consisted of half-cylinders of equal size $(a=3 \mathrm{~mm})$, but they were randomly arranged on different levels, from 0 to $5.5 \mathrm{~mm}$, by increments of $0.5 \mathrm{~mm}$. These levels were generated using a sequence of uniformly distributed random numbers. Disorder was essential, as it prevented slipping of entire layers of particles on the upper bed surface, which would have induced artificial erosion conditions. The effects of bed disorder have been addressed in an earlier paper [23].

An obstacle was set at the channel outlet to allow bed formation and prevent full bed erosion. Its height could be adjusted. Because disorder in the bed-particle arrangement was dependent on the number of layers, the height of the obstacle influenced the entrainment rate and thus the solid discharge [31]. In order to promote disorder, we selected an obstacle height of three particle diameters.

\section{Solid and water supplies}

Colored spherical glass beads with a nominal diameter $2 a$ of $6 \mathrm{~mm}$ and a density $\rho_{p}$ of $2500 \mathrm{~kg} / \mathrm{m}^{3}$ (provided by Sig- 
TABLE I. Flow characteristics and time-averaged values of dimensionless numbers characterizing bed load and water flow. The slope is kept constant: $\tan \theta=10 \%$, while the solid discharge at the inlet $\dot{n}_{0}$ is altered. The notation E10-6 means $\tan \theta=10 \%$ and $\dot{n}_{0} \approx 6$ beads/s. The measured solid discharge within the observation window is denoted by $\dot{n}$. Re, Fr, and $N_{\text {Sh }}$ are the Reynolds, Froude, and Shields dimensionless numbers. The time-averaged particle velocity in the rolling (saltating, respectively) regime is denoted by $\bar{u}_{r}$ $\left(\bar{u}_{s}\right.$, respectively), while $n_{r}\left(n_{s}\right.$, respectively) represents the mean number of rolling (saltating, respectively) particles; the variance (Var) of $n_{r}$ and $n_{s}$ is provided. We have also reported the autocorrelation time $t_{e}$ of the rolling-particle number $n_{r}(t)$.

\begin{tabular}{|c|c|c|c|c|c|c|}
\hline Experiment & E10-6 & E10-7 & E10-8 & E10-9 & E10-16 & E10-21 \\
\hline $\tan \theta(\%)$ & 10.0 & 10.0 & 10.0 & 10.0 & 10.0 & 10.0 \\
\hline$\dot{n}_{0}($ beads/s $)$ & 5.3 & 6.7 & 8.0 & 10.0 & 15.4 & 20.0 \\
\hline$q_{w} / W\left(10^{-3} \mathrm{~m}^{2} / \mathrm{s}\right)$ & 4.15 & 4.42 & 5.38 & 5.54 & 8.19 & 10.31 \\
\hline$h(\mathrm{~mm})$ & 10.2 & 10.6 & 12.2 & 12.3 & 16.6 & 19.1 \\
\hline $\bar{u}_{f}(\mathrm{~m} / \mathrm{s})$ & 0.41 & 0.42 & 0.44 & 0.45 & 0.49 & 0.54 \\
\hline$\dot{n}$ (beads/s) & 5.72 & 6.85 & 7.74 & 9.41 & 15.56 & 20.57 \\
\hline $\mathrm{Re}$ & 4020 & 4090 & 4550 & 4570 & 5280 & 5910 \\
\hline Fr & 1.29 & 1.29 & 1.28 & 1.30 & 1.22 & 1.25 \\
\hline$N_{\mathrm{Sh}}$ & 0.113 & 0.120 & 0.135 & 0.139 & 0.188 & 0.216 \\
\hline$C_{s}(\%)$ & 2.40 & 2.69 & 2.50 & 2.96 & 3.30 & 3.47 \\
\hline $\bar{u}_{r}(\mathrm{~m} / \mathrm{s})$ & 0.063 & 0.074 & 0.065 & 0.075 & 0.075 & 0.072 \\
\hline $\bar{u}_{s}(\mathrm{~m} / \mathrm{s})$ & 0.28 & 0.29 & 0.29 & 0.29 & 0.32 & 0.32 \\
\hline$n_{r}$ & 7.29 & 6.92 & 10.37 & 9.94 & 16.65 & 26.69 \\
\hline $\operatorname{Var}\left(n_{r}\right)$ & 59.13 & 32.72 & 55.82 & 42.61 & 69.37 & 119.06 \\
\hline$n_{s}$ & 2.17 & 2.93 & 3.39 & 3.74 & 6.19 & 7.52 \\
\hline $\operatorname{Var}\left(n_{s}\right)$ & 2.40 & 2.87 & 3.14 & 3.30 & 4.88 & 5.44 \\
\hline$t_{e}(\mathrm{~s})$ & 0.34 & 0.36 & 0.23 & 0.22 & 0.20 & 0.18 \\
\hline
\end{tabular}

mund Lindner GmbH, Germany) were used. They were injected from a reservoir into the channel using a wheel driven by a direct current motor and equipped with 20 hollows on the circumference, as depicted in Fig. 4. For the experiments presented here, the injection rate $\dot{n}_{0}$ ranged from 5 to 20 beads per second, with an uncertainty of less than $5 \%$. This corresponded to a solid discharge per unit width $q_{s} / W$ of $9-38 \times 10^{-5} \mathrm{~m}^{2} / \mathrm{s}$. The water supply at the channel entrance was controlled by an electromagnetic flow meter provided by Krohne (France). The discharge per unit width $q_{w} / W$ ranged from 4 to $10 \times 10^{-3} \mathrm{~m}^{2} / \mathrm{s}$.

The hydraulic conditions (velocity profile, bed friction, etc.) have been specified in earlier papers $[23,32]$. Although

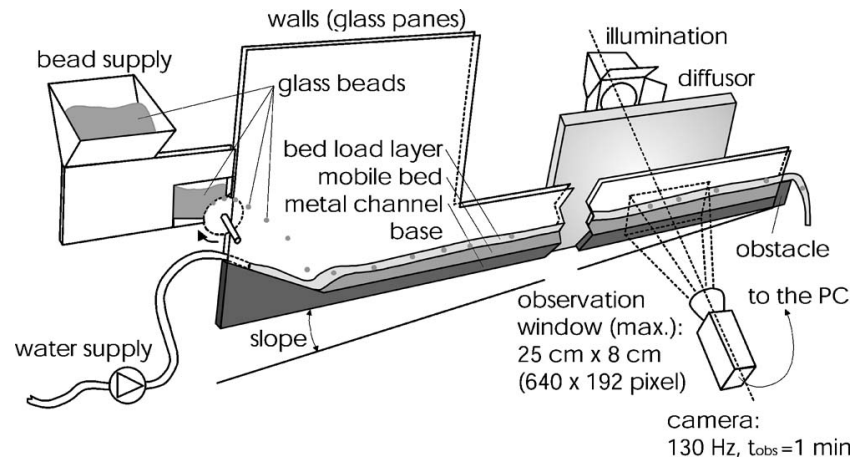

FIG. 4. Sketch of the experimental setup. the flume was narrow, its hydraulic characteristics were similar to those observed in wide channels provided that the flows were shallow. Moreover, making the solid discharge equation dimensionless and comparing it with other laboratory data showed that the features of sediment transport in our flume were quite comparable with those observed with other flumes, although we used spherical particles as well as a narrow and steep channel [33].

\section{E. Experimental procedures}

The experimental procedure can be split into three major steps. First of all, a particle bed was built along the channel base, which remained stationary on average. To that end, an equilibrium between the water discharge, solid discharge, bed elevation, and channel slope was sought. This equilibrium was reached using the following procedure:

(1) The water discharge $q_{w}$ was set to a constant value.

(2) An obstacle (approximately $20 \mathrm{~mm}$ in height) was positioned at the downstream end of the channel. The solid discharge $\dot{n}_{0}$ at the channel entrance (or the injection rate) was set to a constant value. The solid discharge $q_{s}$ was calculated by the relation $q_{s}=\pi d^{3} \dot{n}_{0} / 6$. The first beads supplied by the feeding system were stopped by the obstacle at the channel outlet and started to form a bed. The bed line rose to the level of the obstacle and beads began to leave the channel. After approximately 10 minutes, the system arrived at 
bed-load equilibrium, i.e., there was no more bed deposition or erosion over a sufficiently long time interval.

(3) In order to make the bed line parallel with the channel base, the water discharge was then adjusted. After several iterations, we arrived at the configuration of a bed that consisted of two to three almost stationary bead layers along the channel, for which the bed line slope matched the channel base inclination. Average equilibrium conditions were sustained over long time periods, typically as long as 30 minutes.

Once bed equilibrium was reached, the particles and the water stream were filmed using a Pulnix partial scan video camera (progressive scan TM-6705AN). The camera was placed perpendicular to the glass panes at $115 \mathrm{~cm}$ away from the channel, approximately $80 \mathrm{~cm}$ upstream from the channel outlet. It was inclined at the same angle as the channel. Lights were positioned in the backside of the channel. An area of $L=22.5 \mathrm{~cm}$ in length and $8 \mathrm{~cm}$ in height was filmed and later reduced to accelerate image processing. Refer to the EPAPS document [25] to obtain a short video of run E10-8.

The camera resolution was $640 \times 192$ pixels for a frame rate of $f=129.2 \mathrm{fps}$ (exposure time: $0.2 \mathrm{~ms}, 256$ gray levels). Each sequence was limited to 8000 images due to limited computer memory; this corresponded to an observation duration of approximately 1 minute.

Each experiment was repeated at least twice in order to spot possible experimental problems and to get an idea of the data scattering.

\section{F. Image processing}

Images were analyzed using the WIMA software, provided by the Traitement du Signal et Instrumentation laboratory in Saint-Etienne (France). Positions of the bead mass centers were detected by means of an algorithm combining several image-processing operations. It compared the filmed images with the image of a model bead and calculated the correlation maxima to obtain the bead positions. The water free surface (averaged in the direction perpendicular to the channel walls) was detected using its slim form; missing portions were interpolated or extrapolated. Since the experiments involved a mobile bed, the water depth was defined as the difference between the free surface and the bed surface elevation. Arbitrarily, we considered that the bed surface profile is the broken line linking the top points of the uppermost resting or rolling beads. Figure 5 depicts such a broken line at a given time. Determining the particle states is trickier [23]. Conflicts in the algorithmic state determination arose because distinguishing between incipient motion and small drift (particle oscillations, slight displacement induced by collisions, etc.) was difficult. In our statistical analysis, we considered a fourth population of particles (undeterminate state) that we do not describe here; this detail must, however, be kept in mind when looking at Table I since a small deviation between the computed solid discharge, on the one hand, and the number of moving particles and their velocities, on the other hand, can be observed. For more details, the reader can refer to Refs. $[34,35]$.

\section{EXPERIMENTAL RESULTS}

As we shall see below (Sec. IV A), the generalized Einstein theory predicts a number of features such as the nearly

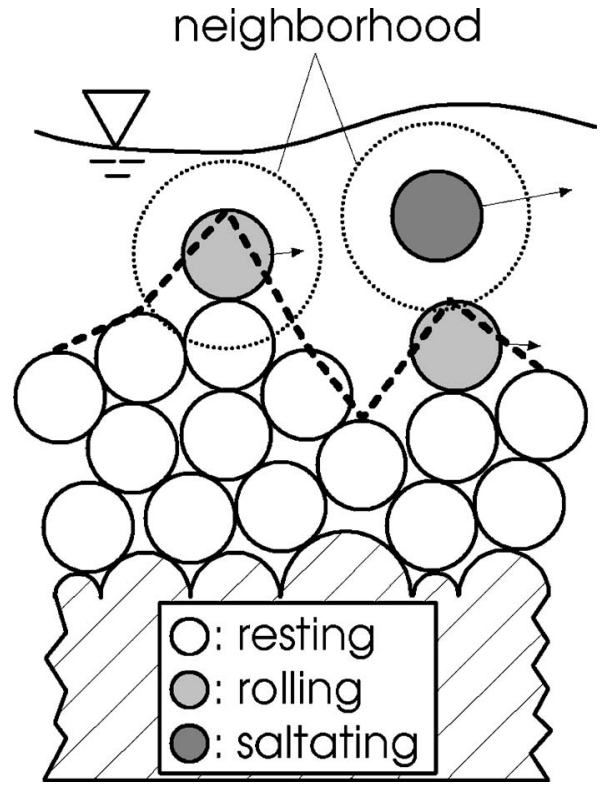

FIG. 5. Sketch defining the state of motion and the bed line.

Gaussian distribution of the solid discharge, the Poissonian character of the occurrence of entrainment and/or deposition over time, and the exponential decrease in the autocorrelation function of the number of particles moving within the observation window. There are also a number of features that conflict with the fundamental assumptions underpinning this theory. In Sec. IV B, we will see that the probability distributions of the key variables have much thicker tails than expected. This will be interpreted as the hallmark of cooperation processes between particles when they are entrained or when they move.

\section{A. Solid-discharge time series}

Figure 6 shows the time variations in the solid discharge $\dot{n}$, the number of particles $n_{r}$ in a rolling regime, the number of particles in a saltating regime $n_{s}$, the number of particles that passed from a resting state to a rolling state $(r \rightarrow b)$ and conversely $(b \rightarrow r)$, and the number of particles that experienced rolling and/or saltating transitions $(r \leftrightarrow s)$. This diagram represents the results obtained for a mean bed slope of 0.1 and a solid discharge at the flume inlet $\dot{n}_{0}=8$ beads $/ \mathrm{s}$ (experiment 10-8 in Table I); these plots are typical of the results that we obtained for other solid discharges $\dot{n}_{0}$.

Note that in these state transitions [see Figs. 6(d) and 6(e)], more than one particle can be involved; because of the limitation of the acquisition rate of our high-speed camera (130 images per second), we could not resolve two events that occurred over very short time intervals. This limitation may pose problems when interpreting the Markovian properties of our time series (see below in this section).

A striking point in Fig. 6 is the wide fluctuations that all the time series exhibit. Typically, the solid flow rate ranged from 0 to 22 beads/s, while the mean flow rate imposed at the inlet was $\dot{n}_{0}=8$ beads $/ \mathrm{s}$. For the rolling regime, the fluctuation range was $0-40$ beads within the observation window, whereas the mean number was $\bar{n}_{r}=9.7$ beads. 

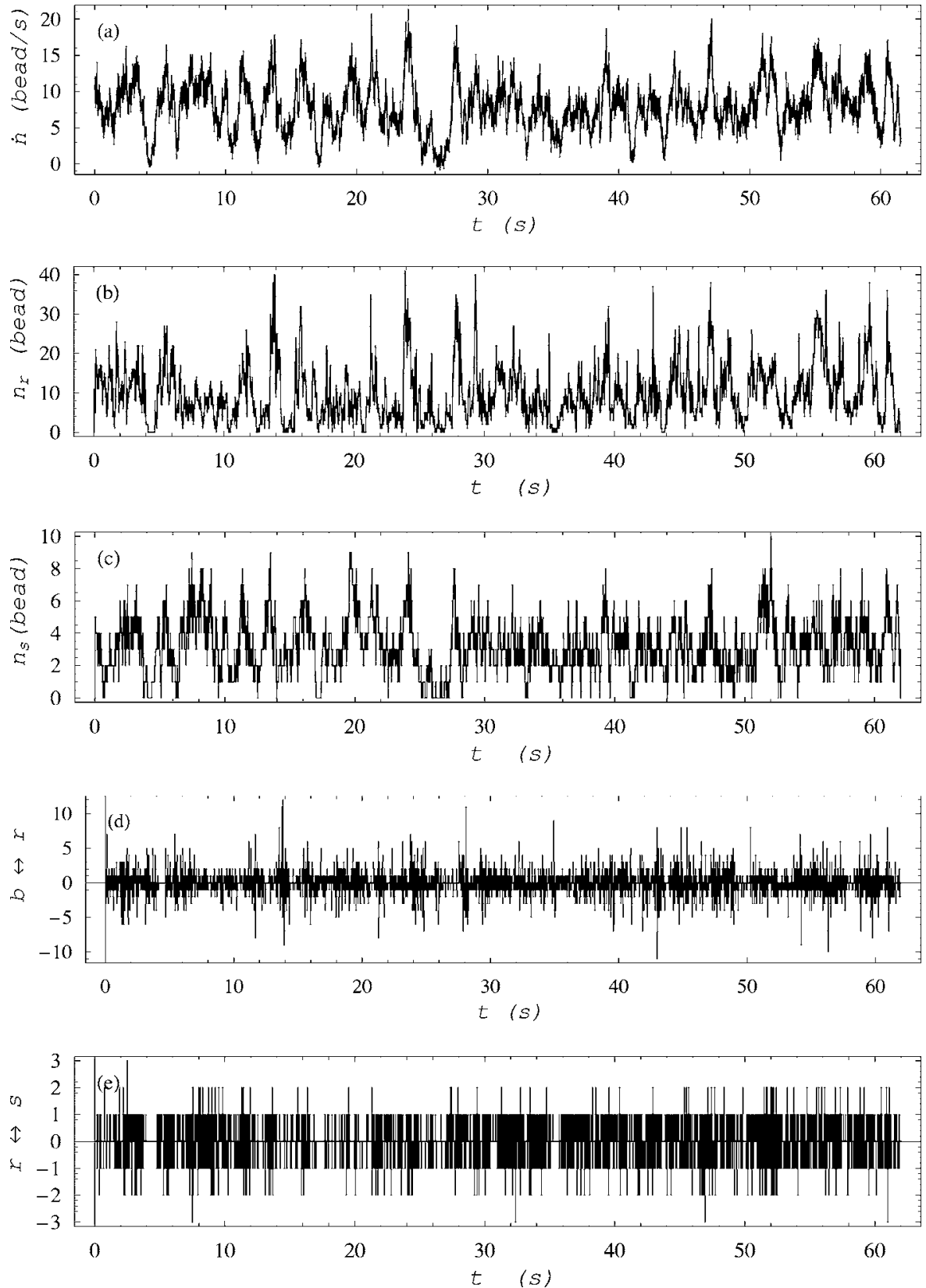

FIG. 6. Experiment 10-8: mean solid discharge at the channel inlet $\dot{n}_{0}=8$ beads/s; mean bed slope $\tan \theta=0.1$. (a) Solid discharge $\dot{n}$ as a function of time. (b) Variation in the number of rolling particles $n_{r}$. (c) Variation in the number of saltating particles $n_{s}$. (d) Exchanges between the bed and the rolling phases: each bar oriented upward indicates the number of beads that passed from the resting state to the rolling regime over a given time interval $\Delta \approx 1 / 130 \mathrm{~s} ;$ downward-oriented bars represent the number of rolling particles coming to a halt. (e) Exchanges between the rolling and saltating regimes.
The origins of these fluctuations have been partly discussed in an earlier paper [23]. In that paper, we tried to understand the origins of the fluctuations by altering particle arrangement within the bed. It was shown that the soliddischarge fluctuations resulted, to a large extent, from the finite size of the observation window. Since the solid discharge was computed using Eq. (3), it was directly related to particle velocities and thus inherited their stochastic variability. It was also observed that these fluctuations were strong compared to experiments run with a stationary bed (i.e., deposition and entrainment of particles were not possible). Here we are attempting to gain insight into the role played by fluctuations in the sediment-transport processes by keeping the same particle arrangement within the bed, but varying the water flow rate while maintaining bed equilibrium (neither net erosion nor deposition over long time scales).
As shown in Fig. 7(a), the empirical probability distribution of solid discharge is closely approximated by a Gaussian distribution, although, in places, there are spikes departing from the Gaussian trend. These spikes reflect the existence of a finite number of particles within the observation window [23]. This Gaussian behavior is expected since the solid discharge is defined as the product of the number of moving particles and of their velocities [see Eq. (3)]. Indeed, if the particle velocities are sufficiently agitated (resulting in a random velocity distribution) and the number of moving particles within the observation window varies significantly with time, the law of large numbers supports this expectation.

As expected, the autocorrelation functions $\rho$ of the measured signals $\dot{n}(t), n_{r}(t)$, and $n_{s}(t)$ are similar. As shown by Fig. 7(b), the typical behavior is the same: (i) we observe a 

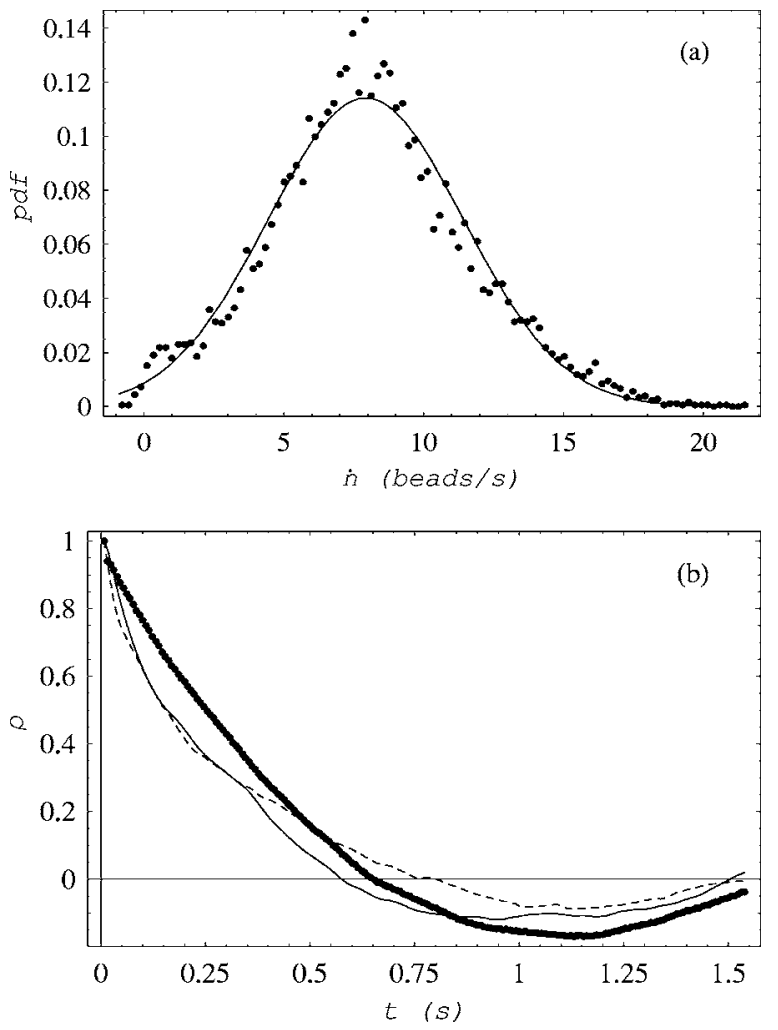

FIG. 7. (a) Probability distribution function (pdf) of the solid discharge for experiment E10-8 $\left(\dot{n}_{0}=8\right.$ beads/s; mean bed slope $\tan \theta=0.1$ ): the dots represent the empirical pdf, whereas the solid line is a Gaussian distribution adjusted on the data (mean, 7.93; standard deviation, 3.49). (b) Autocorrelation function for experiment E10-8: the thick dotted line corresponds to the solid discharge $\dot{n}$, the thin solid line to the number of rolling particles $n_{r}$, and the dashed line to the number of saltating particles $n_{s}$.

fairly slow exponential decrease, i.e., for short times, we have $\rho(s) \approx \exp \left(-t / t_{e}\right)$ with $t_{e}$ a typical time scale; (ii) the typical time scales $t_{e}$ related to each signal are very close. For experiment E10-8, we found $t_{e} \approx 230 \mathrm{~ms}$. The autocorrelation times for other experiments are reported in Table I.

In our earlier paper [23], the time scale $t_{e}$ was interpreted as the typical travel time of the moving particles through the observation window. While this interpretation seems reasonable for the solid discharge, there is at first glance no clear reason why this should be so for the number of rolling and/or saltating particles. One could call on the following explanation for the similarity in the autocorrelation functions: once a particle experiences a transition into another regime, it moves at approximately the same velocity as the mean phase velocity and hence one expects that the autocorrelation time $n_{r}(t)$ and $n_{s}(t)$ is somehow related to a travel time. However, since their mean phase velocity was quite different (see Table I), their autocorrelation times should also be different.

In Sec. II B, we have also shown that for particle entrainment, the waiting-time distribution should follow an exponential distribution of rate $r=N /(\sigma+\tau)$. A particular problem encountered here in evaluating the parameter $r$ is that we could not resolve successive events when they occurred within a very short time interval (less than the acquisition
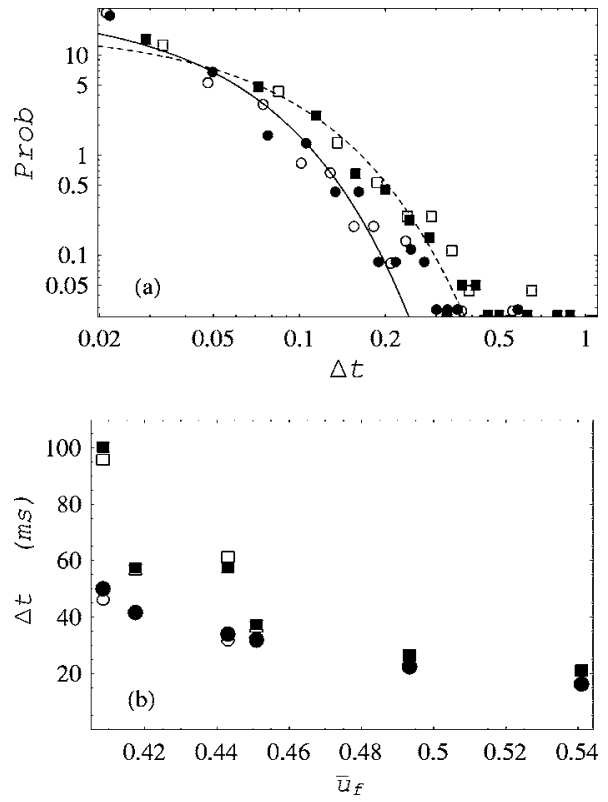

FIG. 8. (a) Probability distribution of the time lag $\Delta t$ between two events (change in state): the filled disks represent the state transition $b \rightarrow r$ (entrainment, $\tau_{b \rightarrow r}$ ), while the empty disks represent the state transition $r \rightarrow b$ (deposition, $\tau_{r \rightarrow b}$ ); the solid line provides the exponential probability distribution adjusted on the data $b \leftrightarrow r$ (using the method of moments). The filled boxes represent the state transition $r \rightarrow s$, while the empty boxes represent the converse transition $s \rightarrow r$; the dashed line is the exponential probability distribution adjusted on the data $r \leftrightarrow s$. (b) Variation in the lag times $\Delta t_{b \rightarrow r}$ (filled disks), $\Delta t_{r \rightarrow b}$ (empty disks), $\Delta t_{r \rightarrow s}$ (filled boxes), and $\Delta t_{s \rightarrow r}$ (empty boxes).

rate of our camera, i.e., for lag times shorter than $2 / 130$ $=0.015 \mathrm{~s}$ ); this means that we should censor the lag-time sample to remove the lowest values if we want to properly evaluate the sample distribution. This also implies that we cannot demonstrate the Markovian character of the measured random variables and this explains why in Fig. 6(e), we can occasionally observe several events occurring at the same time (in contradiction with a Markovian theory).

Within the Eulerian framework adopted in Sec. II B, we referred to an event as any instance where the number of moving particles is changed within the observation window. Figure 3 shows how the step-shaped character is inherited from the individual changes of state. Experimentally, we counted the number of particles that were entrained within the time interval $(1 / 130=7 \mathrm{~ms})$ during which one image was captured by the camera; we did the same for the number of particles that were deposited or experienced a transition to a saltating regime. In doing so, we obtained a time sequence of events, as shown in Figs. 6(d) and 6(e). In this context, the lag time is just the amount of time separating two events of the same type. This lag time is a random variable. Figure 8(a) shows the empirical probability distribution function of the lag times $\Delta t$ for the different classes of events (entrainment or deposition, transition to a rolling or a saltating regime). We have superimposed the exponential distribution, the coefficient of which has been adjusted using the method of moments on the whole sample. As expected, the exponen- 

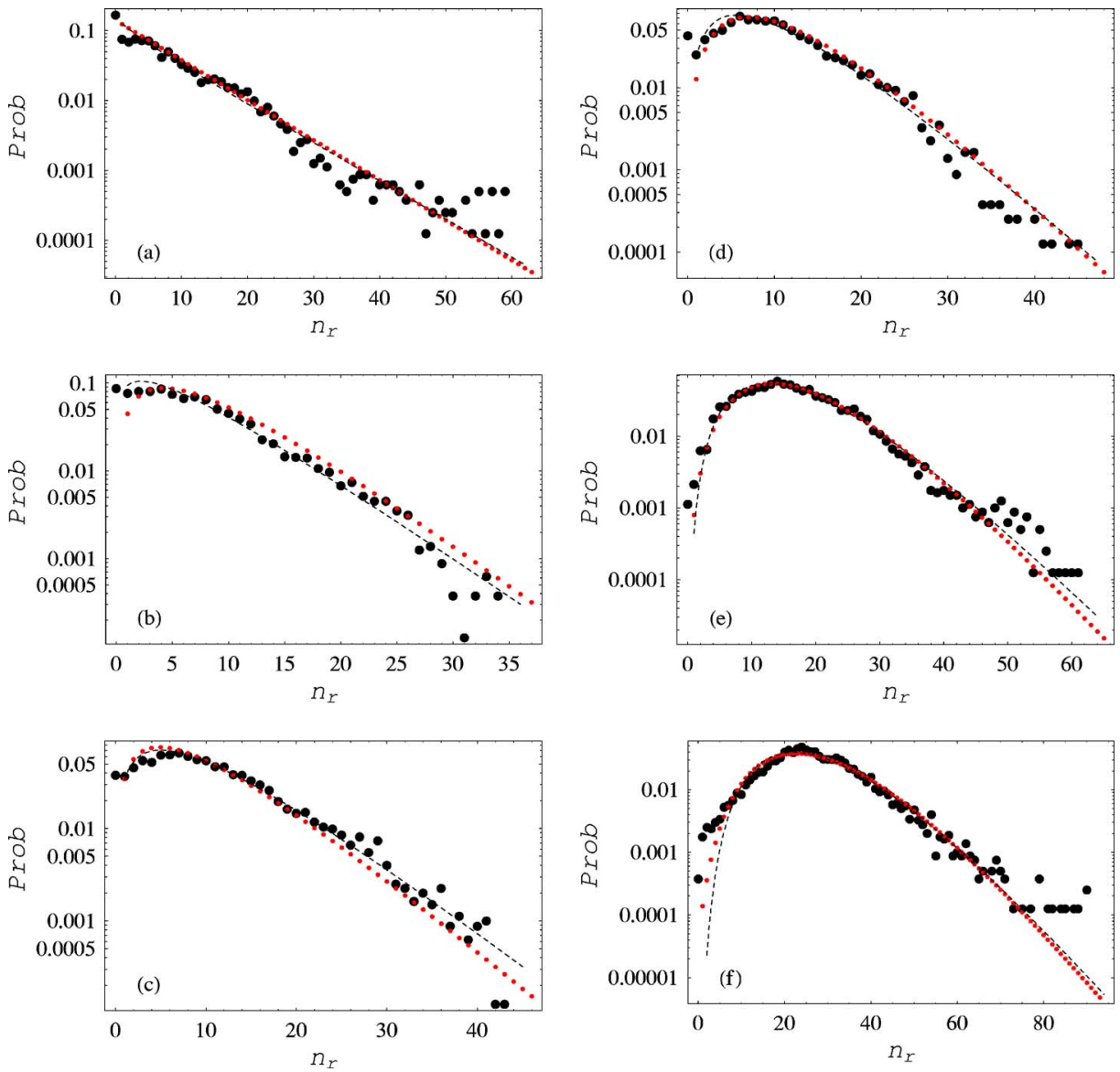

FIG. 9. (Color online) Probability distributions of the number of rolling particles. The dots represent the empirical probability mass functions. The dotted lines represent the negative binomial distribution, while the dashed lines represent the gamma distribution. (a) Experiment E10-6, (b) experiment E10-7, (c) experiment E10-8, (d) experiment E10-9, (e) experiment E10-16, (f) experiment E10-21. tial distribution is a fairly good representation of the lag-time distribution whatever the type of exchange except at low values of $\Delta t$, for which the empirical distribution departs significantly from the exponential trend. Adjustment provides the following characteristic times for each transition type for experiment E10-8: $\Delta t_{b \rightarrow r}=33.9 \mathrm{~ms}$, $\Delta t_{r \rightarrow b}=31.7 \mathrm{~ms}, \Delta t_{r \rightarrow s}=57.4 \mathrm{~ms}$, and $\Delta t_{s \rightarrow r}=61.3 \mathrm{~ms}$. For all experiments, the mean lag times are reported as a function of the mean fluid velocity in Fig. 8(b).

Up to this point, the generalized Einstein theory is qualitatively consistent with our laboratory experiments. A discrepancy is, however, noticeable. In Sec. II B, we found that the autocorrelation time $t_{e}$ was $\tau_{*}=\sigma \tau /(\tau+\sigma) / N$ and the waiting time was $\Delta t_{b \rightarrow r}=r^{-1}=(\sigma+\tau) / N$. From these relations, we deduce that the ratio

$$
\frac{\tau_{*}}{\Delta t_{b \rightarrow r}}=\frac{\tau / \sigma}{(\tau / \sigma+1)^{2}}<1
$$

is in contradiction with our experimental results, since for instance for E10-8, we have $\tau_{*} / \Delta t_{b \rightarrow r}=6.9$. The autocorrelation time is much longer than expected. As we shall see in the next section, this result is not fortuitous and illustrates the existence of long-range correlations in the physical processes governing sediment transport.

\section{B. Probability distribution of the number of moving particles}

Analyzing the probability distribution of the number of moving particles is richer than examining that of the solid discharge because the latter combines two sources of fluctuations: the number of particles and their velocities, which makes it difficult to properly interpret them. Here, we will focus on the probability distributions of the number of rolling particles $n_{r}$ (see Fig. 9) and moving particles $n_{m}$ (see Fig. 10); "moving particles" refer to all particles in motion (in a rolling or a saltating regime). However, given the similarities between the two categories of particles, we will only comment on the former group.

Figure 9 shows how the probability distribution of $n_{r}$ changes when the fluid velocity is increased. At low fluid velocities, the probability distribution is close to a straight line in a log-linear diagram, revealing an exponential behavior. At higher fluid velocities, the probability distribution takes the shape of an asymmetric bell, with its maximum moving from left to right. At first sight, the prominent impression is that increasing the solid discharge leads to making the probability distribution of $n_{r}$ more Gaussian.

In the generalized Einstein theory presented in Sec. II B, we inferred that the number of moving particles should be distributed according to a binomial law, with mean $\bar{n}=\xi N$ and variance $\xi(1-\xi) N$ where $N$ is the density number of particles lying on the bed and $\xi=\tau /(\sigma+\tau)$ is the mean relative time during which a particle is maintained in motion by the stream. A particularity of the binomial law is that its variance must be lower than its mean. For all our experiments, we found that the sample variance exceeded the sample mean. For instance, for experiment E10-8 [see Fig. 9 (c)], the mean number of particles is $\bar{n}_{r}=10.4$, whereas the 

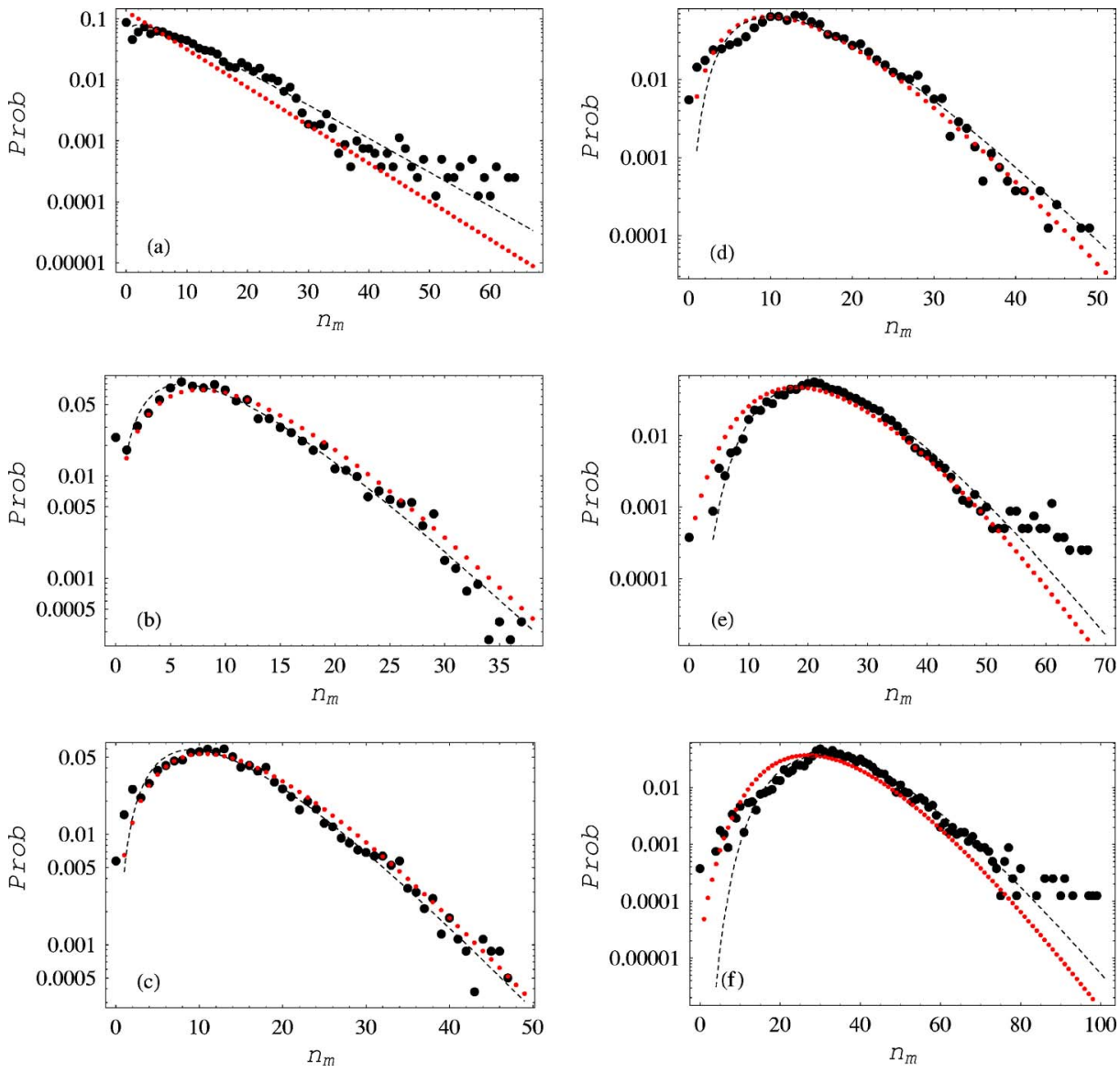

FIG. 10. (Color online) Probability distributions of the number of moving particles. The dots represent the empirical probability mass functions. The dotted lines represent the negative binomial distribution, while the dashed lines represent the gamma distribution. (a) Experiment E10-6, (b) experiment E10-7, (c) experiment E10-8, (d) experiment E10-9, (e) experiment E10-16, (f) experiment E10-21. variance is $\operatorname{Var}\left(n_{r}\right)=55.8$. For all probability distributions, the distribution tail is much thicker than expected.

Since thick tails are often associated with collective phenomena [24], it is worthwhile characterizing these distributions more accurately. We found that the negative binomial distribution provides a fairly proper representation of the empirical distribution, as shown in Fig. 9. Small departures are observed in the distribution tail (insufficient number of data) and when $n_{r} \rightarrow 0$. Note that it was not always very easy to distinguish between incipient motion and oscillations of bed particles (see Sec. III F) and consequently our imageprocessing algorithm failed at times to count the exact number of moving particles. The small deviations between the theoretical and empirical probability distributions may result from this uncertainty on $n_{r}$. Except for the behavior close to the boundaries, the whole trend is well represented by the negative binomial distribution. Instead of a discrete distribution, we can use a continuous probability distribution to approximate the empirical distribution of $n_{r}$. A natural candidate is the gamma distribution, which can be fairly well adjusted on data, as shown in Fig. 9.

This observation is of fundamental importance since it conflicts with the assumptions underlying the birth-and-death process used in the theoretical derivation (see Sec. II B). Indeed, if the particles are independent and identical, then one obtains a binomial distribution whatever the model taken for rest and/or move, provided that the flow is steady and there is bed equilibrium. The only way to obtain a nonbinomial behavior would be to have (i) unsteady flow conditions or (ii) nonidentical or dependent particles.
Concerning point (i), it should be remembered that the negative binomial distribution $\operatorname{Neg}(k \mid r, p)$ can be seen as a Poisson distribution $\operatorname{Po}(k \mid \chi)$, the rate coefficient $(\chi)$ of which is not constant, but randomly distributed according to a gamma distribution $\Gamma\left(\cdot \mid r, p^{-1}-1\right)$

$$
\operatorname{Neg}(k \mid r, p)=\int_{0}^{\infty} \operatorname{Po}(k \mid \chi) \Gamma\left(\chi \mid r, p^{-1}-1\right) d \chi .
$$

This boils down to assuming that the bead supply is not constant, but randomly distributed. This is in contradiction with the boundary conditions that we imposed at the channel inlet.

Point (ii)—nonindependence particles-is physically more convincing. In our experimental observations, we observed that particles preferably moved in groups and over a very short time interval; many particles can be entrained because the dislodgement of one particle entailed the loss of stability of several neighboring particles. These observations may be made clearer by examining the spatiotemporal bead propagation [i.e., by reporting the particle movement in the $(x, t)$-plane]. Figure 11 shows the bead propagation, including a sketch of how the diagram was obtained. Only beads in saltation (black lines) and rolling (gray lines) are presented; beads at rest have been omitted for the sake of clarity. Beads entered the observation window on the left and exited on the right. The time propagation is downward. Since the $x$ component of the velocity of a bead is the ratio between the $x$ displacement and the elapsed time, it is equivalent to the 


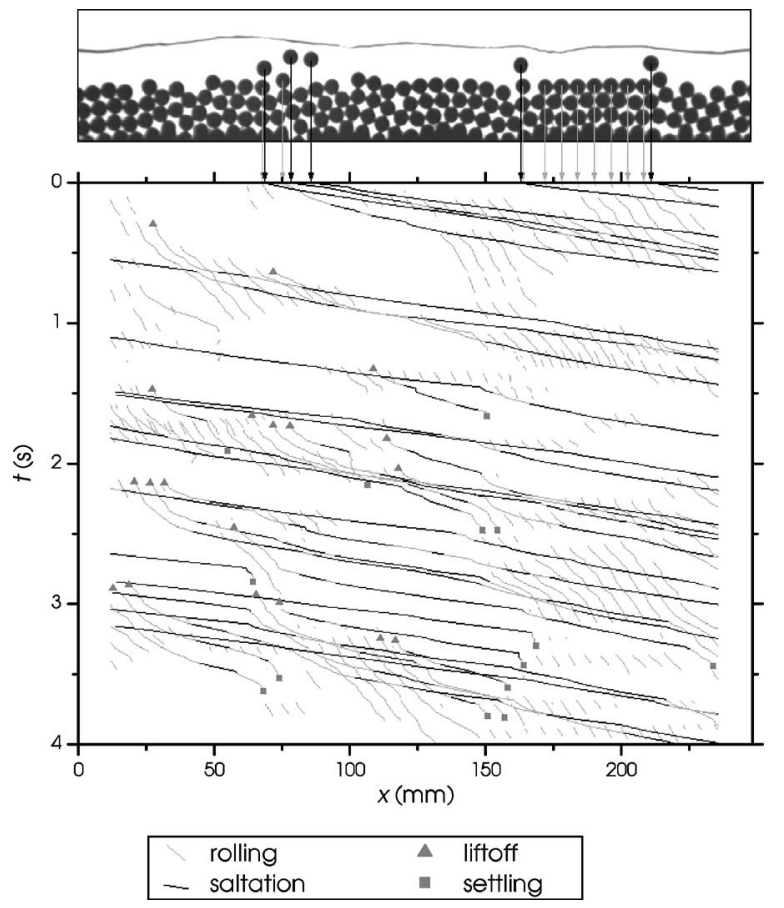

FIG. 11. From the image sequence to the bead propagation in the plane $(x, t)$. Experiment E10-8. We marked the transition from rest to rolling (respectively, from rolling to rest) of the liftoff events (respectively, the settling events) by triangles (respectively, squares). See Ref. [23].

slope of the curve. Given that beads usually moved faster in the saltating regime than in the rolling regime, the $\Delta x / \Delta t$ quotient was larger, as shown in the diagram. Note as well that the beads in saltation typically travelled distances as long as the window length before coming to rest, whereas beads in the rolling regime often moved only a few bead diameters before stopping again.

Furthermore, different events (at a certain $x$ position and a certain time; see Fig. 11) could be observed, for example,

(i) a series of four particles was set into rolling movement (see Fig. 11 at $x \approx 150 \mathrm{~mm}, t \approx 0.4 \mathrm{~s}$ ).

(ii) A bead in saltation was decelerated suddenly because of a collision with another bead. It switched into a rolling regime but returned to saltation a few images later. The bead that had been hit moved about one diameter then came back to rest (see Fig. 11 at $x \approx 150 \mathrm{~mm}, t \approx 1.5 \mathrm{~s}$ ).

(iii) The diagram shows that there were beads frequently switching between rest and rolling; others were switching between the rolling and the saltating regimes, whereas the transition from rest to saltation via rolling (or from saltation to rest) was rare.

(iv) A striking feature is that the liftoff and settling events often occurred in tight ranges of $x$. We observed, for example, seven liftoffs in the range of $10 \mathrm{~mm}<x<40 \mathrm{~mm}$ in the time period $0 \mathrm{~s}<t<3 \mathrm{~s}$ and eight settling events in the range of $140 \mathrm{~mm}<x<170 \mathrm{~mm}$ in the period $1.5 \mathrm{~s}<t<4 \mathrm{~s}$.

Note that, in the diagram of Fig. 11, we show only $4 \mathrm{~s}$ of the sequence, whose total duration exceeded $60 \mathrm{~s}$. The events cited were nevertheless typical of the experiment and reproduced with modifications over the whole sequence. Statisti- cal tests were carried out to determine the relations between cooperative motion (or dislodgement), the bed configuration (i.e., how the particles are arranged in the close neighborhood), and the flow conditions (i.e., triggering mechanisms such as particle collision). Analyzing the image sequences allowed us to formulate different scenarios, but we failed to find any statistical relevance for these scenarios.

\section{CONCLUDING REMARKS}

In this paper, idealized experiments of sediment transport were presented and analyzed in light of Einstein's theory. In the 1940s, Einstein developed a heuristical stochastic theory on sediment transport, based on the recognition that at low fluid velocities, particle transport is highly intermittent [19]. Following the interpretation primarily proposed by Lisle et al. [26], we revisited Einstein's original formulation by stressing the key physical ingredients used in his derivation; we notably showed that describing entrainment and deposition as a birth-and-death process made it possible to retrieve Einstein's bed-load equation and to predict a number of features concerning the probability distributions of the key variables. This statistical description turns out to be especially helpful when testing the reliability of Einstein-type theories: indeed, given the complexity of the physical processes involved, these theories use ad hoc parameters, which must be adjusted. Because of this fitting, any direct comparison against data is biased to a large extent. Moreover, this statistical description may reveal a number of important hidden facets in the physics of sediment transport. For instance, a key assumption in sediment-transport theories is that at low fluid velocities, particles act independently, i.e., the state of a single particle is little or not influenced by other particles. With this assumption, we showed that the probability distribution of the number of moving particles should be binomial and tend quickly toward a Gaussian distribution in the largenumber limit.

Our experimental results provided evidence that, although some statistical properties (such as the autocorrelation function of the solid discharge) predicted by Einstein's theory were consistent with our data, the autocorrelation functions of the number of moving particles and their mass distribution functions violated the assumptions underpinning Einstein's theory. Typically, the autocorrelation time was much longer than expected and the mass distribution function had a much thicker tail than predicted using Einstein's arguments. For instance, from the theoretical standpoint, the number of moving particles within any observation window is a random number distributed according to a binomial distribution; in the large-number limit, the theoretical distribution should tend very quickly toward a Gaussian limit. In contrast, our experiments showed that the sample variance outweighed the sample mean and a negative binomial distribution fits the data better. This means that extreme events (i.e., a large number of moving particles) are much more frequent than expected. Furthermore, our experiments showed that the convergence toward the Gaussian limit is slow. At the lowest solid discharges achievable with our system, the probability distribution of the particle number is closer to an exponential 
distribution. When increasing the solid discharge, the variance and/or mean ratio decreases and the probability distribution becomes increasingly bell shaped.

The discrepancy between theory and experiment revealed the existence of long-range correlations in particle motions. Taking a closer look at the resting and moving states showed that on many occasions, particles moved in well-separated groups. Collective displacement and entrainment of particles explained why the particles were to some extent dependent and thus why the autocorrelation time was much longer than expected.

One might think that aggregate transport was promoted by particle sphericity and equal size. Our observations are, however, well supported by field measurements, which documented similar processes in gravel-bed rivers [16]. The wide range of fluctuations exhibited by laboratory or field measurements [1-3] also confirmed the existence of thick-tailed probability distributions for sediment transport involving irregular particles.

The present study has many important implications. First, it provides a plausible explanation about the failure of all mean-field theories on bed-load transport (at low water discharges), which ignore any cooperation effects between particles. It thus motivates further research with a clear focus on collective effects in entrainment and displacement of coarse particles as a result of fluid action. Second, our investigation has shown the transition to a non-Gaussian behavior toward a Gaussian behavior in the moving-grain population; this transition may explain why mean-field theories successfully describe the water and/or solid discharge relations at high water discharges, while they fail at low water discharges. A striking point is that cooperation turned out to be of great influence when little particles were in motion, but of decreasing strength when the number of particles was increased. Third, this work sheds some light in the critical issues concerning bed-load measurement in rivers [20]. Hydraulicians and geomorphologists use various systems (Helley-Smith sampler, bed-load trap) to measure the solid discharge by capturing sediment over a given time interval. The crux of the issues lies in the proper selection of the sampling time (ranging from a few seconds to several minutes), and this difficulty of selecting a proper time scale is illustrated by the large differences among various measurement systems.

\section{ACKNOWLEDGMENTS}

This study was supported by the Cemagref and funding was provided by Programme Avenir of Rhône Alpes Région, by the Programme national risque naturels of INSU/CNRS, by the Programme national risque hydrologique INSU/ ECCO, and Action Concertée Incitative "Risques naturels" of the CNRS. The authors are grateful to the laboratory TSI (Christophe Duccotet, Nathalie Bochard, Jacques Jay, and Jean-Paul Schon). The work was also supported by specific funds provided by EPFL. The authors thank Professor Anthony Davison (EPFL) for his help in the statistical interpretation of their data.

\section{APPENDIX: EINSTEIN'S ORIGINAL FORMULATION}

\section{Derivation}

In Einstein's theory, the bed-load discharge $q_{s}$ is defined implicitly as the imbalance of entrainment and deposition rates, $\widetilde{E}$ and $\widetilde{D}$, respectively,

$$
\frac{\partial q_{s}}{\partial x}=\widetilde{E}-\tilde{D} .
$$

Under steady and uniform flow conditions, the discharge gradient vanishes and $\widetilde{E}=\widetilde{D}$; Einstein used this equality to compute the solid discharge by assuming that the deposition rate $D$ is an implicit function of the solid discharge [19], as we shall see.

Einstein considered a window of length $L=2 \lambda a$, through which he observed sediment transport. $\lambda$ is a free parameter that will be specified below. The deposition rate $D$ is defined as the number of particles deposited per unit time within this window,

$$
D=\frac{q_{s}}{v_{p}},
$$

where $v_{p}=4 \pi a^{3} / 3$ is the particle volume. The number of particles eroded is related to the probability $p$ that a stationary particle is entrained by the stream over a given time interval $t_{c}$. Since there are approximately $N=2 \lambda a /(2 a)=\lambda$ particles lying at the top of the bed within the window, Einstein defined the entrainment rate per unit time as

$$
E=\lambda \frac{p}{t_{c}}
$$

According to Einstein, the proper time scale $t_{c}$ is related to the particle sedimentation velocity $u_{s}: t_{c}=k a / u_{s}$, where $k$ is a constant parameter. $\mathrm{He}$ also explained that the window length $L$ must be selected so that it corresponds to the mean of the probability distribution of the distances travelled by the moving particles until they are deposited. He considered that this probability distribution is related to the entrainment probability $p$ as follows. Let us consider that $n$ particles are entrained; the mean distance travelled by a particle is denoted by $\ell$. After travelling the distance $\ell,(1-p) n$ are deposited while $p n$ are not deposited. Among these particles, $p(1-p) n$ are deposited after traveling the distance $2 \ell$, but $p^{2} n$ are still in motion, and so forth. The mean deposition distance is then $2 \lambda a=\sum_{n=0}^{\infty}(1-p) p^{n} \ell=\ell(1-p)^{-1}$. From these considerations and the balance $E=D$, he deduced that

$$
q_{s}=\frac{p}{1-p} \frac{\ell}{2 a} \frac{u_{s}}{k a} v_{p}=\frac{1}{k} \frac{p}{1-p} \frac{\ell v_{p}}{2 a^{2}} u_{s} .
$$

\section{Comments}

First, as pointed out by Lisle et al. [26], Einstein implicitly considered that bed-load transport results from alternating periods of motion and rest with a typical time scale of motion that remains very short compared to the resting time. In short, in Einstein's approach, solid discharge does not de- 
pend on particle velocity, but only on the rate at which grains are entrained from the bed. At approximately the same time as Einstein, Kalinske [36] derived a bed-load equation from particle velocity, but without determining the number of particles in motion. Surprisingly enough, it turns out that the two major ingredients used here (the particle velocity and the number of particles in motion) were already recognized 60 years ago as important when taken separately, but their combination seems to have been overlooked.

Second, Einstein introduced the inverse of the settling velocity $u_{s}$ of the particle as the characteristic time $t_{c}$, but without really providing support for this. A number of papers then elaborated on Einstein's formulation and proposed different expressions for the typical time scale $t_{c}$. For instance, instead of $t_{c} \propto a\left(u_{s}\right)^{-1}$, Paintal [37] and Cheng [38] proposed a different definition of the resting time or exchange time $t_{c} \propto a\left(p u_{*}\right)^{-1}$, with $u_{*}$ the shear velocity, which results in a markedly different dependence of the solid charge on $p$. Paintal [37] found that $\dot{n} \propto p^{3}(1-p)^{-1}$ versus $\dot{n} \propto p^{2}(1-p)^{-1}$ for Cheng [38].
Third, in Einstein's approach, the entrainment rate is $E$ $=p N u_{s} /(k a)$, where $N$ is the density number of particles available at the bed surface. This definition implies that at high fluid velocities, the entrainment rate tends toward a constant because the exceedance probability $p$ comes close to unity, which does not make sense. This shortcoming has been seen as a failure of Einstein's approach in computing the solid discharge for any flow rate [39]. In fact, this shortcoming stems from the ad hoc assumptions used by [19], who considered that the features of particle motion remain constant whatever the flow conditions, which contrasts with observations [32]. Note also another obvious limitation of Einstein-type models. At high fluid velocity, the exceedance probability comes close to unity, which causes a nonphysical divergence of the solid discharge in Eq. (A1). In fact, although bed/stream exchanges are the key ingredient of bedload transport for weakly intense flow conditions, they are of decreasing importance with increasing fluid velocity because the bed-load transport takes the form of sheet flows with continuous particle motion $[40,41]$.
[1] P. Wilcock, Earth Surf. Processes Landforms 26, 1395 (2001).

[2] Y. Martin, Geomorphology 53, 75 (2003).

[3] J. Barry, J. Buffington, and J. King, Water Resour. Res. 40, W10401 (2004).

[4] R. Bagnold, Proc. R. Soc. London, Ser. A 405, 369 (1986).

[5] Y. Martin and M. Church, Earth Surf. Processes Landforms 25, 1011 (2000).

[6] R. Bagnold, Professional paper 422-I, U.S. Geological Survey, 1966.

[7] R. Bagnold, Proc. R. Soc. London, Ser. A 332, 473 (1973).

[8] P. Wiberg and J. Smith, J. Hydraul. Eng. 115, 101 (1989).

[9] P.-Y. Julien, Erosion and Sedimentation (Cambridge University Press, Cambridge, 1994).

[10] R. Fernandez Luque and R. van Beek, J. Hydraul. Res. 14, 127 (1976).

[11] Y. Niño, M. Garcìa, and L. Ayala, Water Resour. Res. 30, 1907 (1994).

[12] G. Seminara, L. Solari, and G. Parker, Water Resour. Res. 38, 1249 (2002).

[13] J. Nelson, R. Shreve, S. McLean, and T. Drake, Water Resour. Res. 31, 2071 (1995).

[14] N. Balmforth and A. Provenzale, in Geomorphological Fluid Mechanics, edited by N. Balmforth and A. Provenzale (Springer-Verlag, Berlin, 2001), pp. 369-393.

[15] A. Grass, J. Hydr. Div. 96, 619 (1970).

[16] T. Drake, R. Shreve, W. Dietrich, and L. Leopold, J. Fluid Mech. 192, 193 (1988).

[17] Y. Niño and M. Garcìa, Hydrolog. Process. 12, 1197 (1998).

[18] M. Schmeeckle and J. Nelson, Sedimentology 50, 279 (2001).

[19] H. Einstein, Technical Report No. 1026, United States Department of Agriculture, 1950.

[20] K. Bunte and S. Abt, Water Resour. Res. 41, W11405 (2005).

[21] R. A. Kuhnle and J. B. Southard, Water Resour. Res. 24, 247 (1988).

[22] T. Lisle, Water Resour. Res. 25, 1303 (1989).
[23] T. Böhm, C. Ancey, P. Frey, J.-L. Reboud, and C. Ducottet, Phys. Rev. E 69, 061307 (2004).

[24] D. Sornette, Critical Phenomena in Natural Sciences (Springer, New York, 2000).

[25] See EPAPS Document No. E-PLEEE8-74-092607 for a short movie showing the motion of a few particles in our flume. For more information on EPAPS, see http://www.aip.org/pubservs/ epaps.html.

[26] I. Lisle, C. Rose, W. Hogarth, P. Hairsine, G. Sander, and J. Y. Parlange, J. Hydrol. 204, 217 (1998).

[27] A. Papanicolaou, P. Diplas, N. Evaggelopoulos, and S. Fotopoulos, J. Hydraul. Eng. 128, 369 (2002).

[28] C. Gardiner, Handbook of Stochastic Methods (SpringerVerlag, Berlin, 1983).

[29] C. Ancey, F. Bigillon, P. Frey, and R. Ducret, Phys. Rev. E 67, 011303 (2003).

[30] N. Marsh, A. Western, and R. Grayson, J. Hydraul. Eng. 130, 616 (2004).

[31] M. Dufresne, DEA report, Université Louis Pasteur, 2005.

[32] C. Ancey, F. Bigillon, P. Frey, J. Lanier, and R. Ducret, Phys. Rev. E 66, 036306 (2002).

[33] C. Ancey, T. Böhm, P. Frey, M. Jodeau, and J.-L. Reboud, in River, Costal, and Estuarine Morphodynamics, edited by G. Parker and M. Garcìa (Balkema, Urbana Champaign, 2005), Vol. 1, pp. 3-11.

[34] T. Böhm, C. Duccotet, C. Ancey, P. Frey, and J.-L. Reboud, Exp. Fluids 41, 1 (2006).

[35] T. Böhm, Ph.D. thesis, Joseph Fourier University (2005).

[36] A. Kalinske, Trans., Am. Geophys. Union 28, 615 (1947).

[37] A. Paintal, J. Hydraul. Res. 9, 527 (1971).

[38] N. Cheng, Adv. Water Resour. 27, 937 (2004).

[39] E. Laursen, J. Hydr. Div. 84, 1530 (1958).

[40] B. Sumer, A. Kozakiewicz, J. Fredsoe, and R. Deigaard, J. Hydraul. Eng. 122, 549 (1996).

[41] J. Jenkins and H. Hanes, J. Fluid Mech. 370, 29 (1998). 